The Leitch-Smith model for chondrule formation is at present skeletal, but their demonstration that essentially pristine enstatite chondrites exist is very important. It encourages those (for example ref.16) who are attempting to study chemical variations among chondrules in these meteorites. It should also encourage other workers to move into this neglected corner of chondrite research.

Evidence that the chondrules in carbonaceous, ordinary and enstatite chondrites tell quite different stories illustrates very well the marked change in view alluded to at the beginning of this article. Daly ${ }^{1}$ interpreted chondrites as samples of a planetary mantle and thus, by implication, chemically and physically differentiated materials. Had he proved to be correct, we might now know more than we do about the interior of our planet, but we would surely know much less about its origin. A shattered tenth planet is emotionally appealing, but it would tell us much less about the Solar System's history than 20 or more smaller objects caught at various stages of internal evolution. That the meteorite parent bodies were numerous and varied might disappoint Daly, but it is very good news for planetary scientists.

1. Daly, R.A. Bull. geol. Soc. Am.54, 401 (1943).

. Wasson,J.T. \& Wetherhill, G.W. in Asteroids (ed. T. Gehrels) 926 (1979).

3. Scott, E.R.D. Geochim. cosmochim. Acta 42 , 1447 (1978)

. Dodd, R.T. Meteorites: A Petrologic-Chemical Synthesi. (1981).

Stolper, E., McSween, H.Y. Jr \& Hays, J.F. Geochim. cosmochim. Acta 43, 589 (1979).

Clayton, R N. A Rev. nucl, particle Phys, 28, 501 (1978)

7. Larimer, J.W. \& Anders, E. Geochim. cosmochim. Acto 34, 367 (1970)

8. Wasson, J.T. Meteorites, Classification and Properties (1974).

9. Dodd, R.T. Geochim. cosmochim. Acta 33, 161 (1969)

10. Heyse, J.V. Earth planet Sci. Lett. 40, 365 (1978).

11. Hutchison, R., Bevan, A.W.R., Agrell, S.O.\& Ashworth, J.R. Nature 280, 116 (1979)

12. McSween, H.Y. Jr Geochim. cosmochim. Acta 41, 1843 (1977)

13. Dodd, R.T. Earth planet Sci. Lett. 40, 71 (1978).

14. Van Schmus, W.R. \& Wood, J.A. Geochim. cosmochim. Acta 31, 747 (1967)

5. Leitch, C.A. \& Smith, J.V. Nature 283, 60 (1980).

16. Denham, M. Personal communication (1981).

\title{
Why is the moon slowing down?
}

\section{from David W. Hughes}

THE Moon orbits the Earth every month and on average moves $13.2^{\circ}$ per day across the sky. As long ago as 1695 Edmond Halley (Phil. trans. R. Soc. 19, 1960; 1695) suspected that the Moon was slowing down in its orbit. Now, that the effect has been confirmed, two main causes have been proposed: tidal dissipation and variation in the value of $\mathrm{G}$, the universal 'constant' of gravitation. Measurements of this acceleration have been reviewed and interpreted by F.R. Stephenson of the Department of Geophysics, Liverpool University in a recent edition of the Journal of the British Astronomical Association (91, 136; 1981).

Tidal friction retards the Earth's rotation. The length of the day is thus slowly increasing at a rate of about one second in 50,000 years. As an autonomous system conserves its angular momentum, the angular momentum lost by the Earth is gained by the Sun and Moon. The Sun is so massive that the reciprocal effect on it is negligible but the Moon does, however, gain a significant amount of angular momentum at the expense of the Earth. This causes our satellite to drift away at a rate of about $4 \mathrm{~cm}$ per year.

The acceleration of the Moon can be calculated in two independent ways. The first relies on the analysis of ancient eclipse observations which extend back over 2,000 years. The difference between the predieted lunar position, if we neglect acceleration, and the observed position which obviously is affected by the acceleration, increases as the square of the elapsed time. Over 2,000 years this difference is as large as two or three degrees. The most recent result obtained using this technique is $-30 \pm 3$ arc sec century ${ }^{2}$. An indirect method based on the observations of the transits of Mercury since 1743 produced a result of $-26 \pm 2$ arc sec century ${ }^{2}$.

The second approach is through the study of oceanic tides. Recent research has shown that the sea level has not varied by more than a few metres over the last three millennia and there is no evidence of any significant change in the lunar tidal friction over the past 2,500 years. It has also been found that the vast open oceans are the major energy sink and not, as previously thought, shallow regions such as the Bering Sea. Calculations of the mean rate of work done by the Moon on the ocean surfaces resulted in an acceleration of $-30.6 \pm 3.1$ arc sec century ${ }^{-2}$. Ocean tides perturb the orbits of Earth satellites and an analysis of these perturbations lead to a result of $-27.4 \pm 3$ arc sec century ${ }^{-2}$.

The recession rate $\mathrm{r}$ is related to the accel- eration $\dot{n}$ and the rate of change of the constant of gravitation $\dot{G}$ by the following equation:

$$
\frac{\dot{\mathrm{r}}}{\mathrm{r}}=-\frac{2 \mathrm{n}}{3 \mathrm{n}}+\frac{1}{3} \frac{\dot{\mathrm{G}}}{\mathrm{G}}
$$

If $\dot{\mathrm{n}}$ is measured and $\dot{\mathrm{G}}$ is assumed to be zero, $\dot{r}$ can be easily calculated. The present value for $\dot{\mathrm{r}}$ is $4.0 \pm 0.3 \mathrm{~cm} \mathrm{yr}^{-1}$. The Moon was once much closer to the Earth than it is now. G.H. Darwin (Scientific Papers 2, 208 , Cambridge, 1908) originally extended this deduction to claim that the Moon may have originated as part of the Earth. The recession rate is inversely proportional to the sixth power of the distance. The assumption that the present rate of energy dissipation has always been active would bring the Moon catastrophically close to Earth less than $1.5 \times 10^{9} \mathrm{yr}$ ago. The Earth is three times older than this. Here, to quote W.H. Munk (Quarterly Journal of the Royal Astronomical Society 9, 352; 1968), "we are extrapolating from a few thousand years to a few thousand million years, always a dangerous undertaking, and a harrowing one when the very process is in doubt". Fortunately it has been shown that even moderate changes in the shape of oceans and adjacent seas may materially affect the tidal dissipation and hence the recession rate. The astronomical approach thus seems to be of little help in solving the problem of exactly where the Moon was in the geological past.

The difference between the acceleration measured as a function of Ephemeris Time and the acceleration measured as a function of Atomic Time yields the nontidal component and consequently the rate of change of $\mathrm{G}$. Some 7,000 observations of lunar occultations of stars have been used by Van Flandern to find an Atomic Time value of the acceleration of $-36 \pm 5$ arc sec century ${ }^{-2}$. Laser reflectors have been also placed on the Moon by Apollo astronauts and have been used to give a lunar acceleration with an Atomic Time value of $-23.8 \pm 4$ arc sec century ${ }^{2}$.

Stephenson carefully combines the Ephemeris Time and Atomic Time values of the acceleration. There seems to be considerable overlap between the two data sets and he concludes that the present observational evidence is quite consistent with the interpretation that $\dot{G}$ is zero and the universal constant of gravitation is not varying.

What will be the end result of lunar recession? The day (the Earth's spin period) and the month (the lunar orbital period) are both increasing, the day faster than the month. Eventually it will take Earth fifty-five of our present days to spin once. It will also take the Moon fifty-five days to orbit. Day and month will be equal and the same part of the Earth's surface will always face the Moon.

David $W$. Hughes is in the Department of Physics, University of Sheffield. 